\title{
Similarities and differences in lectin cytochemistry of laryngeal and tracheal epithelium and subepithelial seromucous glands in cases of sudden infant death and controls
}

\author{
F P Paulsen, T Tschernig, A S Debertin, W J Kleemann, R Pabst, B N Tillmann
}

\begin{abstract}
Background-It has been speculated that non-specific defence mechanisms of the epithelium and subepithelial seromucous glands play a role in the larynx and lungs in cases of sudden infant death.

Methods-The larynx and trachea from five children who had died of sudden infant death (SID) syndrome and five control cases of comparable age were compared for the presence of lectin binding sites (12 different lectins tested).

Results-The secretory product of mucin producing cells contained carbohydrates including galactose and sialic acids. Binding sites for fucose and N-acetylgalactosamine were only present in some of the specimens and distribution revealed no correlation between cases of SID and controls. Epithelial cells and serous cells of seromucous glands contained binding sites for sialic acid in cases of SID and controls. Moreover, binding sites for mannose were detected in these cells but were only present in SID cases. The difference between the SID and control groups as to the presence/expression of concanavalin $A$ was highly significant.

Conclusions-It is suggested that mucus hypersecretion in SID occurs in response to bacterial toxins or viral infection and is not specific. The different binding sites for mannose in cases of SID and controls could indicate differences in the production of antimicrobial peptides. A disturbed expression pattern of antimicrobial peptides in children who later succumb to SID could be responsible for an imbalance of the local microflora with a higher density of microorganisms on the mucosa. Further studies are required to elucidate the pattern of expression of antimicrobial peptides in subsequent SID victims.
\end{abstract}

(Thorax 2001;56:223-227)

Keywords: sudden infant death; mucin; carbohydrate

Infections may play a crucial role in sudden infant death (SID). Many epidemiological risk factors have been identified for SID-for example, viral infections, exposure to cigarette smoke, and winter peak mild respiratory symptoms. ${ }^{1}$ Mild infections of the respiratory tract or, less commonly, of the middle ear, gastro- intestinal tract or other organs occurred in $30-85 \%$ of cases. ${ }^{2}{ }^{3}$ Virus infections and bacterial toxins induce cytokine activity which might have an important role in the immune response of the respiratory tract and be one possible trigger mechanism. ${ }^{4-7}$ For these reasons, delayed or deficient immunological protection, variable competence of cellular and humoral immunological reactions, as well as overstimulation of the mucosal immune system have been considered as possible causative agents in SID. ${ }^{4-10}$

In this context, non-specific defence mechanisms may also be relevant. ${ }^{11-13}$ Recent results suggest that some components of mucus are secreted in excess in some cases of SID. ${ }^{14}$ Hypersecretion of mucus is recognised to be a contributory factor in the morbidity and mortality in chronic airway disease ${ }^{15}$ as it can become a source of disability when the type of mucus glycoprotein is altered and its function is no longer protective.

This study was undertaken to investigate the larynx and trachea in cases of SID and in controls with a view to determining the nonspecific immunological characteristics of the lining epithelium and subepithelial seromucous glands. The binding of 12 different lectins to the epithelium and subepithelial seromucous glands was tested to determine whether they act as indicators for the nonspecific immune response and protection of the epithelia in the two groups.

\section{Methods}

Thorough post mortem examinations were performed at the Institute of Forensic Medicine of the Hanover Medical School on five cases of SID (two girls, three boys) and five controls (two girls, three boys) aged 50-128 days (table 1). Organ samples were routinely obtained during legal necropsies for histopathological examination. The mean age of the SID and control groups was 82 and 81 days, respectively. The control group consisted of two infants with traumatic causes of death (drowning and exsanguination) and three children who died of natural causes (bronchopneumonia, meningitis, and adenovirus infection). SID was defined (according to Beckwith ${ }^{16}$ ) as "sudden death in any infant or young child between seven and 730 days which is unexpected by history, and in which a thorough post mortem examination failed to demonstrate an adequate cause of death". Mild infections of the upper respiratory tract or 
Table 1 Clinical data on children

\begin{tabular}{lll}
\hline Age (days) & Sex & Cause of death \\
\hline 50 & $\mathrm{M}$ & SID \\
60 & $\mathrm{M}$ & SID \\
80 & $\mathrm{~F}$ & SID \\
100 & $\mathrm{~F}$ & SID \\
121 & $\mathrm{M}$ & SID \\
71 & $\mathrm{M}$ & Exsanguination \\
94 & $\mathrm{M}$ & Drowning \\
37 & $\mathrm{~F}$ & Meningitis \\
76 & $\mathrm{M}$ & Bronchopneumonia \\
128 & $\mathrm{~F}$ & Adenovirus infection \\
\hline SID &
\end{tabular}

SID $=$ sudden infant death .

middle ear were not considered to have been the cause of death. Three laryngeal and tracheal sections from the children were excised, fixed in $4 \%$ formaldehyde, and embedded in paraffin.

For analysis by light microscopy, frontal and horizontal sections $(7 \mu \mathrm{m})$ were deparaffinised and stained with toluidine blue $(\mathrm{pH} 8.5)$, alcian-blue $(\mathrm{pH} 1)$, and mild periodate alkaline Schiff stain (mPAS) according to instructions provided by Veh et al. ${ }^{17}$

For lectin binding experiments, $7 \mu \mathrm{m}$ tissue sections were deparaffinised in xylene, rehydrated with a graded series of ethanol, passed into double distilled water, and finally into Tris buffered saline $(0.05 \mathrm{M}$ Tris, $0.15 \mathrm{M}$ sodium chloride, $\mathrm{pH}$ 7.4, TBS) supplemented with $0.02 \mathrm{M} \mathrm{CaCl}_{2}$. Incubation of the sections with various fluorescein isothiocyanate (FITC) conjugated lectin solutions $(10 \mu \mathrm{g} / \mathrm{ml})$ was performed in a dark moist chamber at room temperature for 30 minutes. After washing the slides three times with TBS, sections were mounted in $10 \%$ glycerol in TBS or in Vectashield (Vector Laboratories, Burlingame, CA, USA). The specificity of the lectin binding was checked in control experiments by mixing the lectin solution with the corresponding inhibiting sugar (table 2) at concentrations of
$0.2-0.5 \mathrm{M}$ prior to incubation for 30 minutes at room temperature. The origins and specificities of the lectin conjugates are listed in table 2 . TBS mounted sections were used to estimate the autofluorescence of the tissues. All slides were examined using a microscope equipped for epifluorescence (Zeiss-Axiophot, Oberkochen, Germany).

Two tailed $\mathrm{p}$ values were calculated using the Fisher exact test (SPSS for Windows 6.0.1, SPSS Inc, Chicago, USA).

\section{Results}

LIGHT MICROSCOPY

The tunica mucosa of the larynx and trachea presented a pseudostratified columnar epithelium and a lamina propria containing many seromucous glands. Goblet cells were integrated in the epithelium as solitary cells. The secretory product of the goblet cells as well as mucous parts of the seromucous glands showed strong positive reactions with alcianblue ( $\mathrm{pH} 1)$ and with mild periodate Schiff base (mPAS) in both cases of SID and controls.

SIMILARITIES IN THE LECTIN STAINING PATTERN OF SID AND CONTROLS

Application of carbohydrate specific lectins (Con A, UEA I, GSA I, GSA II, MPA, PNA, Jacalin, WGA, suc-WGA, MAA, LPA, SNA) to deparaffinised tissue sections of laryngeal and tracheal epithelium and lamina propria from children who had died from SID and those who had died from other known diseases (natural death) or lethal trauma resulted in different staining patterns of goblet cells and epithelial cells as well as mucous parts and serous parts of the subepithelial seromucous glands (tables 3 and 4). Binding sites for MPA, Jacalin, suc-WGA, and MAA were uniformly distributed throughout the goblet cells and mucous parts of the subepithelial seromucous glands,

Table 2 Origin and carbohydrate specificities of lectin conjugates used in this study

\begin{tabular}{|c|c|c|c|}
\hline Lectin & Abbreviation & Carbohydrate specificities & Inhibitor \\
\hline Concanavalin A agglutinin & ConA & $\alpha-$ Man & $\alpha$-methyl-Man $+\alpha$-methyl Glc \\
\hline Ulex europaeus I agglutinin & UEA-I & $\alpha$-L-Fuc & Fuc \\
\hline Griffonia simplicifolia I agglutinin & GSA-I & $\alpha-\mathrm{GalNAc}>\alpha-\mathrm{Gal}$ & $\mathrm{GalNac}+\mathrm{Gal}$ \\
\hline Griffonia simplicifolia II agglutinin & GSA-II & $\alpha(\beta)-$ GlcNAc & GlcNAc \\
\hline Maclura pomifera agglutinin & MPA & $\alpha$-GalNAc & GalNAc \\
\hline Arachis hypogaea agglutinin & PNA & D-Gal- $\beta(1-3)$ GalNAc & Gal \\
\hline Artocarpus integrifolia & Jacalin & Gal- $\beta$ (1-3) GalNAc & Gal \\
\hline Wheat germ agglutinin & WGA & $\alpha-$ GlcNAc $>\alpha-$ GalNAc $>$ Neu 5 Ac & GlcNAc, Neu5Ac \\
\hline Succinylated wheat germ agglutinin & sucWGA & $\alpha-$ GlcNAc $>\alpha-$ GalNAc & GlcNAc \\
\hline Maackia amurensis agglutinin & MAA & Neu5Ac $\alpha(2-3)$ Gal & Neu5Ac \\
\hline Limulus polyphemus agglutinin & LPA & $\begin{array}{l}\text { Neu5Ac } \alpha(2-3) \text { GalNAc } \\
>\text { Neu5Ac }(2-6) \text { GalNAc }\end{array}$ & BSM \\
\hline Sambucus nigra agglutinin & SNA & Neu5Ac $\alpha(2-6) \mathrm{Gal}$ & Neu5Ac \\
\hline
\end{tabular}

$\mathrm{BSM}=$ bovine submandibular gland mucin $; \mathrm{Fuc}=$ fucose $; \mathrm{Gal}=$ galactose $;$ GalNAc = N-acetyl-galactosamine; Glc $=$ glucose; Glc$\mathrm{NAc}=\mathrm{N}$-acetyl-glucosamine; Man $=$ mannose $;$ Neu $5 \mathrm{Ac}=\mathrm{N}$-acetyl-neuraminic acid

Table 3 Summary of lectin binding in goblet cells or mucous parts of subepithelial seromucous glands

\begin{tabular}{cllllllllllllll}
\hline & Case no & Con $A$ & UEA I & GSA I & GSA II & MPA & PNA & facalin & WGA & suc-WGAMAA & LPA & SNA \\
\hline SID & 1 & - & - & + & - & + & ++ & +++ & +++ & +++ & + & - & + \\
& 2 & - & +++ & +++ & - & + & - & +++ & +++ & ++ & + & - & + \\
& & - & - & +++ & - & + & - & +++ & +++ & ++ & + & - & + \\
& & - & - & - & - & + & - & +++ & +++ & +++ & + & - & + \\
& Controls & & - & + & +++ & - & + & + & +++ & +++ & ++ & + & - & + \\
2 & - & - & - & - & + & + & +++ & +++ & +++ & + & - & + \\
& & - & ++ & +++ & - & + & + & +++ & +++ & +++ & + & - & + \\
& & - & +++ & - & - & ++ & - & +++ & +++ & ++ & + & - & + \\
& & - & - & - & - & + & - & +++ & +++ & +++ & + & - & + \\
& & - & + & - & - & + & - & +++ & +++ & +++ & + & - & + \\
\hline
\end{tabular}


Table 4 Summary of lectin binding in epithelial cells or serous parts of subepithelial seromucous glands

\begin{tabular}{|c|c|c|c|c|c|c|c|c|c|c|c|c|c|}
\hline & Case no & Con $A$ & $U E A I$ & GSA I & GSA II & $M P A$ & $P N A$ & Facalin & $W G A$ & $\begin{array}{l}s u c- \\
W G A\end{array}$ & $M A A$ & $L P A$ & $S N A$ \\
\hline \multirow[t]{5}{*}{ SID } & 1 & ++ & - & - & - & - & - & - & + & - & - & - & + \\
\hline & 2 & ++ & - & - & - & - & - & - & + & - & - & - & + \\
\hline & 3 & ++ & - & - & - & - & - & - & + & - & - & - & + \\
\hline & 4 & + & - & - & - & - & - & - & + & - & - & - & + \\
\hline & 5 & ++ & - & - & - & - & - & - & + & - & - & - & + \\
\hline \multirow{5}{*}{ Controls } & 1 & - & - & - & - & - & - & - & + & - & - & - & + \\
\hline & 2 & - & - & - & - & - & - & - & + & - & - & - & + \\
\hline & 3 & - & - & - & - & - & - & - & + & - & - & - & + \\
\hline & 4 & - & - & - & - & - & - & - & + & - & - & - & + \\
\hline & 5 & - & - & - & - & - & - & - & + & - & - & - & + \\
\hline
\end{tabular}

whereas epithelial cells were negative for MPA, Jacalin, suc-WGA, MAA, UEA I, GSA I, and PNA (figs 1, 2, and 3). WGA and SNA binding was detected in all goblet cells, epithelial cells, and serous as well as mucous parts of the subepithelial seromucous glands. No staining was seen with GSA II and LPA.

DIFFERENCES IN THE LECTIN STAINING PATTERN OF SID AND CONTROLS

Staining was visible in goblet cells or mucous parts of subepithelial seromucous glands (table 3 ) in a few cases with SID (UEA I, $n=2$; GSA $\mathrm{I}, \mathrm{n}=4$; PNA, $\mathrm{n}=2$ ) and in some of the controls (UEA I, $\mathrm{n}=3$; GSA I, $\mathrm{n}=1$; PNA, $\mathrm{n}=2$ ).

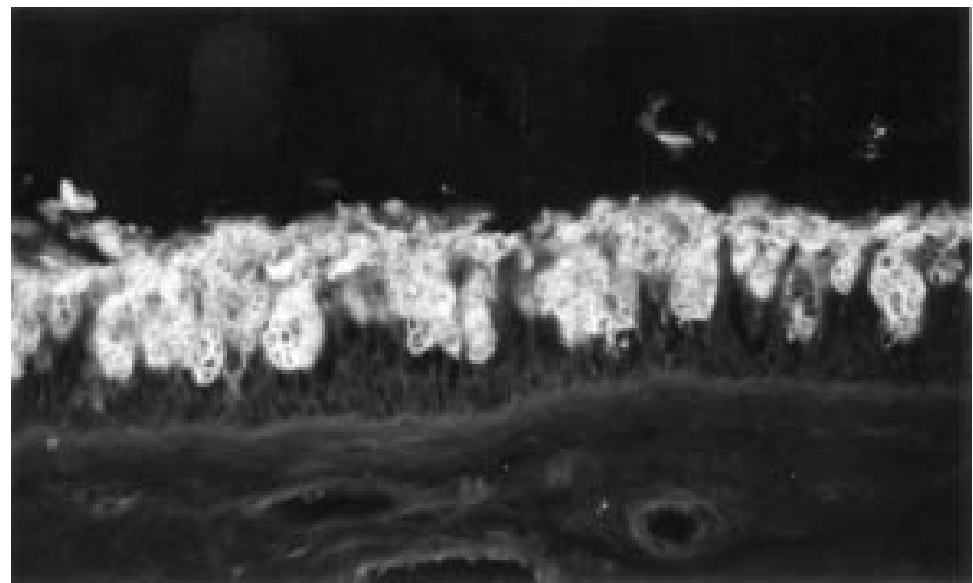

Figure 1 Histochemical staining with the lectin succinylated wheat germ agglutinin (suc-WGA) in a case of sudden infant death (SID). Suc-WGA binding is uniformly distributed throughout the goblet cells whereas the epithelial cells are completely suc-WGA negative.

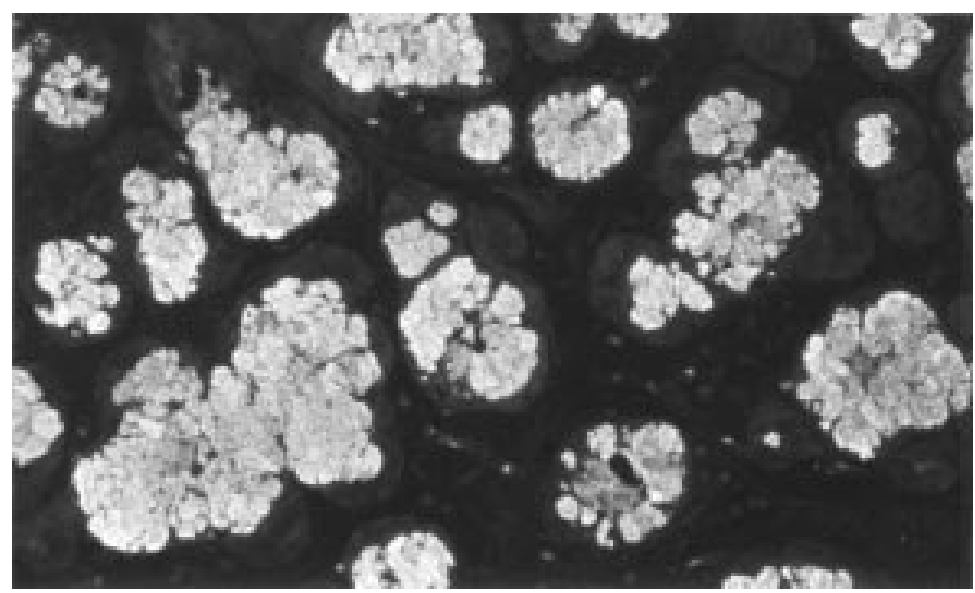

Figure 2 Histochemical staining with the lectin succinylated wheat germ agglutinin (suc-WGA) in a case of sudden infant death (SID). Suc-WGA binding is uniformly distributed throughout the mucous parts of the seromucous glands whereas the serous parts are completely suc-WGA negative.
Interestingly, ConA staining was found only in cases of SID (table 4). In these cases ConA binding was restricted to epithelial cells and to the serous parts of the subepithelial seromucous glands (figs 4 and 5) and did not have binding sites in goblet cells or the mucous parts of the subepithelial seromucous glands (tables 3 and 4). No staining with ConA was seen in any of the controls (table 4). The difference between the two groups was not significant for GSA I in mucous parts of the subepithelial seromucous glands $(p=0.206)$ but was significant for Con $\mathrm{A}$ in the serous parts of the subepithelial seromucous glands $(p=0.008)$.

\section{Discussion}

In this study we used both light microscopy and lectin histochemistry to study the larynx and trachea in cases of SID and in controls with a view to determining the non-specific immunological characteristics of the lining epithelium and subepithelial seromucous glands. We found no differences in the secretory product of goblet and mucous cells of seromucous glands, but there were differences in the binding pattern of epithelial cells and serous cells of seromucous glands between cases of SID and controls.

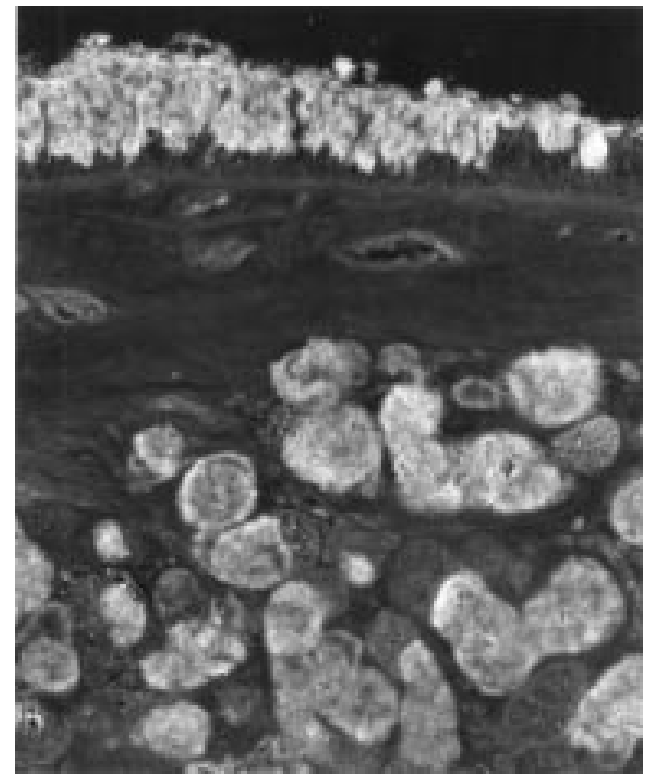

Figure 3 Histochemical staining with the lectin succinylated wheat germ agglutinin (suc-WGA) in a control case. Suc-WGA binding is uniformly distributed throughout the goblet cells in the epithelium and the mucous parts of the subepithelial seromucous glands whereas the epithelial cells and serous parts of the seromucous glands are completely suc-WGA negative. 


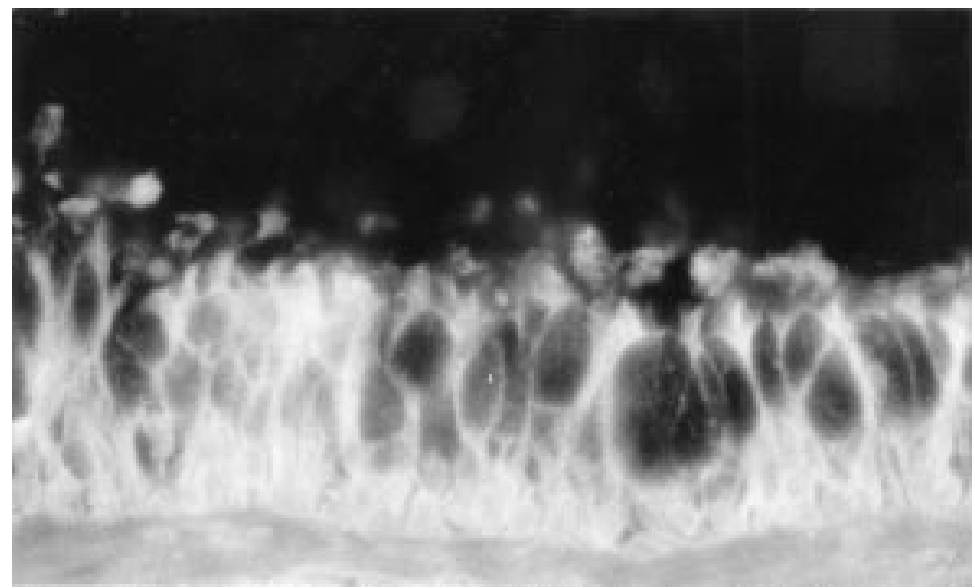

Figure 4 Histochemical staining with the lectin concanavalin A (Con A) agglutinin in a case of sudden infant death (SID). The Con A stain is restricted to epithelial cells and does not appear in the goblet cells.

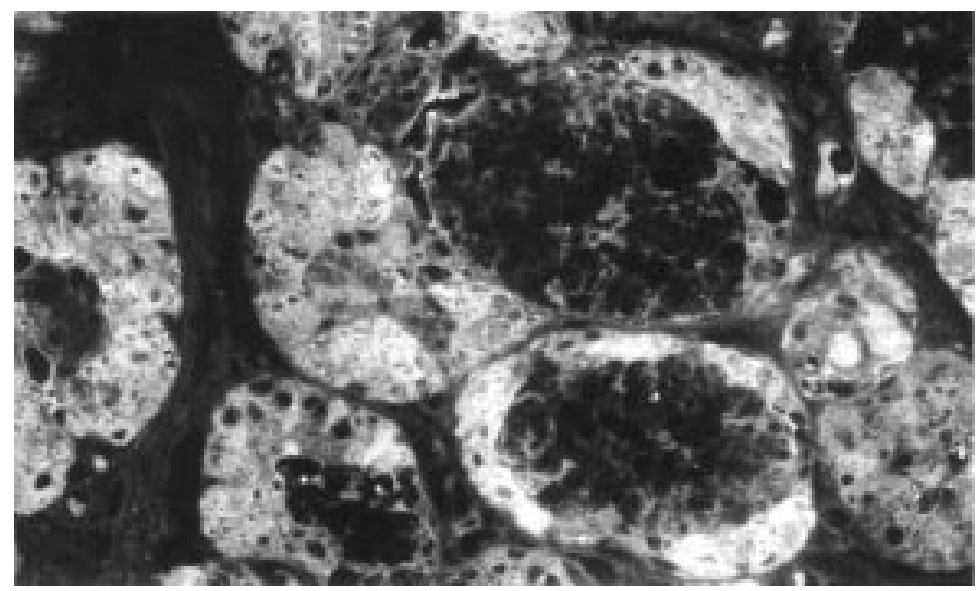

Figure 5 Histochemical staining with the lectin concanavalin A (Con A) agglutinin in a case of SID. The Con A stain is restricted to the serous parts of the seromucous glands and does not appear in the mucous parts.

Mucin producing cells are present in the laryngeal and tracheal epithelium as well as in subepithelial seromucous glands. Our results show that the secretory product of these cells contains carbohydrates including galactose, $\mathrm{N}$-acetyl-glucosamine, and $\mathrm{O}$-acetylated as well as non-O-acetylated sialic acids (table 3 ). Sialic acids are present in $\alpha(2-6)$ linkage in all goblet and epithelial cells as well as in mucous and serous cells of the seromucous glands, whereas $\alpha(2-3)$ linkages are detectable only in goblet and mucous cells of the seromucous glands as shown by SNA and MAA binding, respectively (table 3 ). Binding sites for fucose and $\mathrm{N}$-acetyl-galactosamine were only present in some of the mucin producing cells and distribution at these sites showed no correlation with their presence in cases of SID and controls.

As is known from the gastrointestinal tract, mucus serves several functions. Besides lubricating the mucosa and water proofing to regulate epithelial cell hydration, mucins protect mucosal surfaces against potentially harmful substances such as particles, digestive enzymes, and toxins, as well as bacterial and other infectious agents. ${ }^{18-23}$ It has long been assumed that mucus protects mucosal surfaces from infective agents. Intestinal mucus has been observed to carry away bacteria. ${ }^{24}$ Moreover, it has been shown that mucus possesses structures that mimic the receptor sites for microorganisms on epithelial cells that facilitate trapping and subsequent disposal of bacteria ${ }^{25}$ and viruses. ${ }^{26}$

Mucus hypersecretion has been shown to be present in some cases of SID. ${ }^{11}$ Moreover, Brock $^{14}$ specified this finding and showed that sulphated mucus glycoproteins are secreted in excess in some victims of SID. Our data reveal that there are no significant differences in the quality of the secreted mucin between the SID cases and controls. Goblet cells and mucous cells of subepithelial seromucous glands contain binding sites for fucose and $\mathrm{N}$-acetylgalactosamine in some cases of SID, in addition to binding sites for galactose, $\mathrm{N}$-acetyl-glucosamine, and $\mathrm{O}$-acetylated as well as non-O-acetylated sialic acids. However, this finding was also seen in the control group and does not seem to be specific. A possible tendency should be studied in more cases. Based on our results, we suggest that mucus hypersecretion in SID occurs in response to bacterial toxins or viral infection which have been shown to be a major risk factor. ${ }^{127}$

Interestingly, we found binding sites for mannose to be present in the epithelial cells and serous cells of seromucous glands only in cases of SID. No binding sites for mannose were detected in the control group. This reveals a highly specific difference between the SID and control groups as to the presence/ expression of Con A. We were not able to determine whether this finding was the result of glucose deprivation or to the addition of mannose in our SID cases as is known, for example, from the carbohydrate deficient glycoprotein syndrome (CDGS) type $1 .{ }^{28} \mathrm{On}$ the other hand, it is possible that mannose residues in our controls were masked by other carbohydrates and were therefore not detected, or that glycosylation was lost in the SID cases because of pathogenic events.

It has been shown that multicellular organisms have to survive in an environment populated by numerous, potentially life threatening, microorganisms. Different strategies have been developed to ward off infections by preventing the attack of microorganisms that have already entered the epithelia. It is therefore not surprising that epithelia are equipped with various antimicrobial substances that act rapidly to kill a broad range of microorganisms. There is strong evidence that, in addition to constitutively secreted peptide antibiotics such as lysozyme, lactoferrin and $\alpha$-defensins, others are induced on contact with microorganisms or by proinflammatory cytokines. ${ }^{29}$ The $\beta$-defensins represent one family of vertebrate antimicrobial peptides, members of which are inducible and have recently been identified in humans, especially in the lung. ${ }^{30} 31$ The local pattern of expression characteristic of defensins may indicate that specialised surfaces express a characteristic surface antimicrobial peptide pattern that could in turn define the characteristic microflora as well as the density of microorganisms present on the surface. ${ }^{29}$ Moreover, it has been shown that 
antimicrobial peptides are not only secreted by epithelial cells but also by serous cells of seromucous glands. ${ }^{32} 33$

The different binding patterns of Con A to laryngeal and tracheal epithelium and to subepithelial seromucous glands seen in cases of SID and controls may indicate differences in the production of antimicrobial peptides as has been shown for lysozyme and lactoferrin. ${ }^{34}$ Indeed, antimicrobial peptides containing mannose are known in insects but unfortunately have not been analysed in vertebrates until now. However, a disturbed pattern of expression of antimicrobial peptides in children who later succumb to SID could be responsible for an imbalance in the local microflora with a higher density of microorganisms on the mucosa, which might be responsible for immunological reactions described in cases of SID. Further studies are required to elucidate the expression pattern of antimicrobial peptides, especially those that are inducible such as human $\beta$ defensin-2 (HBD-2), in subsequent cases of SID.

We thank Karin Stengel and Philipp Steven for technical assistance, $\operatorname{Dr} M$ Schlaud for help in statistical evaluation, and Michael Beall for correcting the English.

1 Raza MW, Essery SD, Elton RA, et al. Exposure to cigarette smoke, a major risk factor for sudden infant death syndrome: effects of cigarette smoke on inflammatory responses to viral infection and bacterial toxins. FEMS Immunol Med Microbiol 1999;25:145-54.

2 Berry PJ. Pathological findings in SID. I Clin Pathol 1992;45:11-6.

3 Kleemann WJ, Hiller AS, Tröger HD. Infections of the upper respiratory tract in sudden infant death syndrome. upper respiratory tract in sudden
Int $\mathcal{F}$ Legal Med 1995;108:85-9.

4 Stoltenberg L, Saufstad OD, Rognum TO. Sudden infant death victims show local immunoglobulin $M$ responses in tracheal wall and immunoglobulin $M$ response in duodenal mucosa. Pediatr Res 1992;31:272-5.

5 Blackwell CC, Saadi AT, Raza MW, et al. The potential role of bacterial toxins in sudden infant death syndrome (SID). Int $\mathcal{F}$ Legal Med 1993;105:333-8.

6 Tschernig T, Kleemann WJ, Pabst R. Bronchus-associated lymphoid tissue (BALT) in the lungs of children who had died from sudden infant death syndrome and other causes. Thorax 1995;50:658-60.

7 Hiller AS, Kracke A, Tschernig T, et al. Comparison of the immunohistology of mucosa-associated lymphoid tissue in the larynx and lungs in cases of sudden infant death and controls. Int f Legal Med 1997;110:316-22.

8 Roche WR. Immunopathology in SID. F Clin Pathol (Suppl) 1992;45:46-8.

9 Reid GM, Tervit H. Sudden infant death syndrome (SID): immunoglobulins and hypoxia. Med Hypotheses 1995;44: 202-6.

10 Stoltenberg L, Vege A, Saugstad OD, et al. Changes in the concentration and distribution of immunoglobulinproducing cells in SID palatine tonsils. Pediatr Allergy Immunol 1995;6:48-55.

11 Fink BR, Beckwith JB. Laryngeal mucous gland excess in victims of sudden infant death. Am F Dis Child 1980;134 $144-6$
12 Harrison DFN. Histologic evaluation of the larynx in sudden infant death syndrome. Ann OtoRhinoLaryngol 1991;100:173-5.

13 Harrison DFN. Laryngeal morphology in sudden unexpected death in infants. F Laryngol Otol 1991;105:646-50.

14 Brock CA. An evaluation of mucus glycoproteins in the larynges of victims of sudden infant death syndrome. $\mathcal{7}$ Laryngol Otol 1995;109:403-9.

15 Jany B, Basbaum CB. Mucin in disease: modification of mucin gene expression in airway disease. Am Rev Respir Dis 1991;144:38-41.

16 Beckwith BJ. Observations on the pathological anatomy of the sudden infant death syndrome. In: Bergmann AB, Beckwith JB, Ray CG, eds. International conferences on causes of sudden death in infants. Seattle, London: University of Washington Press, 1970:83-139.

17 Veh RW, Corfield AP, Schauer R, et al. The bovine submandibular gland. 2. Histochemical and biomechanical results. In: Schauer R, Boer P, Buddecke E, Kramer MF, results. In: Schauer R, Boer P, Buddecke E, Kramer MF, Stuttgart: Thieme, 1997:193-4.

18 Failard H, Schauer R. Glycoproteins as lubricants, protective agents, carriers, structural proteins and as participants in other functions. In: Gottschalk A, ed. Glycoproteins. Their composition, structure, and function. 2nd edn, part B. Amsterdam: Elsevier 1972:1246-67.

19 Forstner JF. Intestinal mucins in health and disease. Digestion 1978;17:234-63.

20 Hutch JA. The role of urethral mucus in the bladder defense mechanism. F Urol 1970;103:165-7.

21 Walker WA. Host defense mechanisms in the gastrointestinal tract. Pediatrics 1976;57:901-16.

22 Schauer R. Sialinsäurereiche Schleime als bioaktive Schmierstoffe. Nachr Chem Tech Lab 1992;40:1227-31.

23 Reuter G, Struwe R, Feige J, et al. Analysis of carbohydrate composition and sialidase activity in oral secretions of patients with tumors in the upper aerodigestive tract. Eur Arch Otorhinolaryngol 1992;249:5-11.

24 Florey HW. Observations on the functions of mucus and the early stages of bacterial invasion of the intestinal mucosa. $\mathcal{F}$ Pathol Bacteriol 1933;37:283-9.

25 Abraham SN, Beachey EH. Host defenses against adhesion of bacteria to mucosal surfaces. Host Def Mech 1985;4:6388.

26 Reuter G, Schauer R, Bumm P. Sialic acids of human nasal mucin, possible targets of influenza $C$ viruses. In: Schauer $\mathrm{R}$, Yamakawa T, eds. Sialic acids. Proceedings of the fapanese German symposium on sialic acids. Kiel: Berbel-Mendel, German symposin $1988: 259-60$.

27 Blackwell CC, MacKenzie DA, James VS, et al. Toxigenic bacteria and sudden infant death syndrome (SID): nasopharyngeal flora during the first year of life. FEMS Immunol Med Microbiol 1999;25:51-8.

28 Korner C, Lehle L, Figura v K. Carbohydrate-deficient glycoprotein syndrome type 1: correction of the glycosylaion defect by deprivation of glucose or supplementation of mannose. Glycoconj 7 1998;15:499-505.

29 Schröder JM. Epithelial amtimicrobial peptides: innate local host response elements. Cell Mol Life Sci 1999;56:32-46

30 Bals R, Goldman MJ, Wilson JM. Mouse $\beta$-defensin 1 is a salt-sensitive antimicrobial peptide present in epithelia of the lung and urogenital tract. Infect Immun 1998;66:122532 .

31 Bals R, Wang X, Wu Z, et al. Human beta-defensin 2 is a salt-sensitive peptide antibiotic expressed in human lung. $\mathcal{F}$ Clin Invest 1998;102:874-80.

32 Park K, Lim DJ. Development of secretory elements in murine tubotympanum: lysozyme and lactoferrin immunohistochemistry. Ann OtoRhinoLaryngol 1993;102:385-95.

33 Miyazaki T, Fujiki T, Inoue Y, et al. Immunoelectron microscopic identification of lysozyme-expressing cells in human
labial salivary glands. Arch Histol Cytol 1998;61:199-214.

34 Brandtzaeg P, Halstensen TS, Huitfeldt HS, et al. Epithelial expression of HLA, secretory component (poly-Igreceptor), and adhesion molecules in the human alimentary tract. Ann NY Acad Sci 1992;664:175-9. 